\title{
Electrical stimulation of the lumbrical muscles in an incomplete quadriplegic patient: case report
}

\author{
S G Carroll BAppSc MSc, ${ }^{1}$ S F Bird BAppSc, ${ }^{2}$ D J Brown MBBS FRACP FACRM ${ }^{3}$
}

Departments of ${ }^{1}$ Physiotherapy and ${ }^{2}$ Occupational Therapy, ${ }^{3}$ Spinal Injuries Unit, Austin Hospital, Heidelberg, Victoria, Australia, 3084.

The increasing number of incomplete cervical spinal cord injuries means that more attention needs to be focused on the rehabilitation of the incomplete quadriplegic hand. A case study, describing the application of electrical stimulation for strengthening the paretic lumbrical muscles, is presented. A 2 week strengthening program resulted in a $33 \%$ increase in the force produced by the lumbrical muscles. No loss of strength had occurred 4 weeks after cessation of the treatment. The magnitude and speed of this result should be of interest to those clinicians who seek to maximise patient independence in minimal time.

Key words: electrical stimulation; lumbrical muscles; quadriparesis rehabilitation; spinal cord injuries.

\section{Introduction}

With improvements in the acute management of accident victims with suspected spinal cord injuries, an increasing proportion of patients with incomplete quadriplegia are being seen in rehabilitation facilities. As part of a clinical programme using electrical stimulation to augment voluntary muscle function in spinal cord injured patients, we treated the lumbrical muscles of an incomplete quadriplegic patient and observed rapid improvement in function. We therefore decided to study the next suitable patient and document thoroughly the changes induced by electrical stimulation. We report here on the successful application of electrical stimulation for strengthening the lumbrical muscles in the left hand of the second patient.

\section{Methods}

\section{Case study}

The second patient was a 43 year old man who fractured his sixth cervical vertebrate in a fall which resulted in an incomplete C5-6 quadriplegia. He demonstrated marked weakness in both his upper and lower limbs on admission and was treated conservatively with bedrest in head tongs for 9 weeks. His acute management was otherwise unremarkable.
Status of upper limb recovery

Nine months after his injury, the subject had regained useful function in his left, non dominant hand with good voluntary wrist flexion and extension (equivalent to grades of $4-5^{1}$ ) and some voluntary control of finger flexion and extension, and thumb flexion, extension and abduction (grades $2-3)$. Recovery of the intrinsic function was, however, limited to outer and mid range metacarpophalangeal flexion with interphalangeal extension (grades 1-2). Recovery of the right hand was limited to minimal finger and thumb flexion and extension (grade 1) with no function in the intrinsic muscles. With this level of function, the subject was almost independent in self care but complained of limited strength in the grip of his left hand.

\section{Design of the study}

To examine the feasibility of using electrical stimulation to strengthen paretic lumbrical muscles, a 6 week clinical trial, incorporating repeated measurements, was undertaken. Assessments of muscle strength and active range were performed prior to the commencement of, twice during and twice following a 2 week period of lumbrical stimulation. 


\section{Assessment procedures}

As the lumbrical muscles tend to work as a functional group, rather than for the individual fingers, the maximal voluntary metacarpophalangeal (MCP) flexion force exerted by all 4 lumbrical muscles on a pressure pad (Talley Inc, Borehamwood, Hertfordshire, UK) was measured. Four maximal voluntary efforts were performed and the mean force was calculated. For the duration of this test, all proximal and distal interphalangeal (IP) joints were splinted into extension to prevent any contribution of the finger flexors. In addition, the subject's thumb was immobilised in extension and his wrist was immobilised in the neutral postion to prevent any contribution from these structures.

Active range of the lumbricals was defined as the maximal amount of MCP flexion that could be achieved by each finger without allowing any active IP flexion. The starting position of full MCP extension was defined as being 180 degrees and the subject was asked to "bend his knuckles as far down as he could without letting his fingers bend'. A manual goniometer (Rolyan Medical Products, Menomonee Falls, WI, USA) was used to measure the point at which IP flexion first appeared.

\section{Training programme}

Following completion of the pre-treatment assessment, the subject commenced a programme of stimulation for 30 minutes a day, 5 days a week for 2 weeks, at a duty cycle of 4 seconds on, 6 seconds off. The patient displayed excellent compliance and reported that the stimulation was neither uncomfortable nor tedious. After completion of the treatment period, the subject was discharged home and returned for 2 further reassessments after 2 weeks and then 4 weeks. All assessments were performed by the same 2 therapists (SGC and SFB), using the same equipment and techniques.

\section{Stimulation}

A commercial muscle stimulator (Agar 4000 Neuromuscular stimulator, Ginosar Electronic Products, Kibbutz Ginosar, Israel) which produced a modified square wave of 200 microseconds duration was utilised. The frequency of stimulation was $60 \mathrm{~Hz}$ and the amplitude was set to give maximal MCP flexion without any IP flexion. The patient was encouraged to work actively with the stimulator to produce the desired movement. Two carbon rubber electrodes (Medtronic Inc, Minneapolis, MN, USA) were used. The cathode, measuring $2.5 \times 2.5 \mathrm{~cm}$, was placed proximally over the median nerve at the wrist. The circular anode, measuring $1 \mathrm{~cm}$ in diameter, was positioned between the first and second metacarpal heads on the palm of the hand. This position gave consistent lumbrical responses in the second, third and fourth fingers. Despite considerable manoeuvering of both cathode and anode, a consistent response of the fourth lumbrical, in the fifth finger, could not be obtained. No results are therefore given for this finger.

\section{Results}

Electrical stimulation of the lumbrical muscles produced a $33.5 \%$ increase in MCP flexion force after 2 weeks of treatment (Fig 1). A major proportion of this increase $(27.4 \%)$ had occurred after the first week of treatment with only a $6.1 \%$ increase being recorded after the second week's treatment. Further assessments of MCP flexion force at 2 weeks and at 4 weeks after the cessation of treatment showed that these gains in force were maintained.

Repeated measurements of active range of MCP flexion showed a similar although less consistent trend in the second, third and fourth fingers. Active range of MCP flexion improved by an average of $12.33 \pm 8.1$ degrees after the first week of treatment. Assessment at the conclusion of the treatment period showed that further gains were not achieved although the initial gains were largely maintained (11.33 \pm 6.1 degrees). After 2 weeks without treatment, mean active range had dropped slightly to $10.0 \pm 6.6$ degrees and after a further 2 weeks to $7.33 \pm 7.1$ degrees.

Measurement of passive range of the MCP and IP joints of the left had using a goniometer was also performed at each 


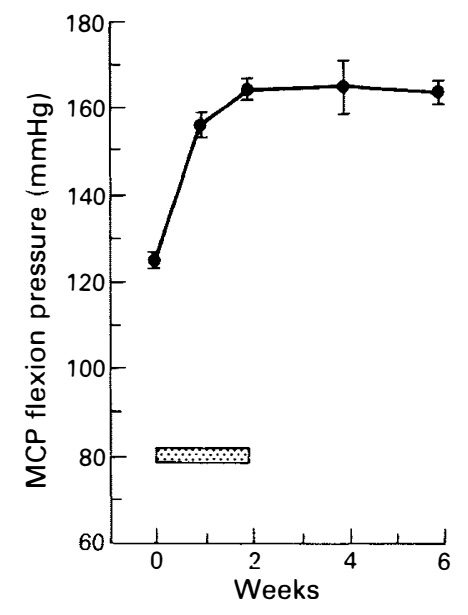

Figure 1 Metacarpophalangeal flexion pressure recorded before, during and after a 2 week period of electrical stimulation (notated by solid bar). Values plotted are the mean and standard errors of 4 attempts.

assessment. Full passive range was achieveable in all joints except for the proximal IP joint of the fourth finger which consistently demonstrated a flexion contracture of 15 degrees.

\section{Discussion}

The first and second lumbrical muscles generally arise from the radial side of the first and second tendons of flexor digitorum profundus respectively. The third and fourth lumbricals arise from adjacent sides of the second and third, and third and fourth flexor digitorum profundus tendons respectively. Each lumbrical muscle tapers to a tendon and inserts into the radial side of the dorsal digital expansion of the associated finger. The discrepancy betweeen the numbering of the fingers and the lumbricals arises because there is no lumbrical muscle associated with the first finger (ie thumb). The eighth cervical and first thoracic nerve roots supply all the lumbrical muscles. The supply to the first and second lumbricals, however, is derived from the median nerve whereas the ulnar nerve typically supplies the third and fourth lumbricals. Instances where the third lumbrical is also supplied via the median nerve are not uncommon. ${ }^{2}$ Functionally, the lumbricals act to flex the MCP joints while maintaining the IP joints in extension by virtue of their insertion into the dorsal digital expansion. They thus play a key role in stabilising the MCP and IP joints during grasping activities. They also assist extensor digitorum to extend the IP joints while the MCP joints are extending. A detailed description of the primary and secondary actions of the lumbrical muscles can be found in Long and Brown. ${ }^{3}$

Two clinically relevant findings have emerged from this case study: (1) that electrical stimulation can be used successfully to strengthen rapidly the paretic lumbrical muscles of an incomplete quadriplegic's hand; and (2) that these gains can be maintained after cessation of treatment. It should also be noted that this effect was achieved by clinical therapists using commercially available equipment. Given that this improvement occurred 9 months after the initial injury, during a period characterised by a lack of progress and when no other hand therapy was being offered, it would be difficult to argue that these results were unrelated to the use of electrical stimulation. It is interesting to note that the gains in active range were not as well maintained as the gains in strength; perhaps reflecting the fact that maximum torque is generated in the mid range, rather than the terminal range of movement.

Our application of this technology differs from that of many others who have focused on the use of electrical stimulation in the upper limb for the restoration of hand function rather than its rehabilitation. For example, some microprocessor controlled, multichannel implantable stimulation systems can now provide a few select quadriplegic individuals with grasping ability. ${ }^{4,5,6}$ To the best of the authors' knowledge, no reports on the successful or otherwise application of electrical stimulation to the intrinsic muscles of the hand have been published. In fact, little attention if any has been paid to the intrinsic muscles of the quadriplegic hand and this is no doubt due to the fact that many victims of cervical spinal cord injury do not regain any finger function at all. For those who do, it is 
typically said that improvement can take up to 2 years. Yet the improvement reported here occurred after only 2 weeks of treatment; a finding, we consider, is of clinical relevance. The fact that this prospective study confirmed our initial observations suggests that this technique might be of value in the management of other patients with similar hand dysfunction. Additional studies of other patients are also warranted.

\section{Acknowledgements}

This study has been assisted by grants from the Austin Hospital Medical Research Foundation and the Physiotherapy Research Foundation.

\section{References}

1 Medical Research Council (1943) Aids to the Investigation of Peripheral Nerve Injuries. War Memorandum No. 7 2nd ed. (revised). His Majesty's Stationary Office, London.

2 Wynn-Parry CB (1973) Rehabilitation of the Hand. 3rd ed. Butterworths, London.

3 Long C, Brown ME (1964) Electromyographic kinesiology of the hand: muscles moving the long finger. $J$ Bone Joint Surg 46A: 1683-1706.

4 Hoshimiya N, Naito A, Yajima M, Handa Y (1989) A multichannel FES system for the restoration of motor function in high spinal cord injury patients: a respiration-controlled system for multi-joint upper extremity. IEEE Trans Biomed Eng 36: 754-760.

5 Kiwerski J, Weiss M, Pasniczek R (1983) Electrostimulation of the median nerve in tetraplegics by means of implanted stimulators. Paraplegia 21: 322-326.

6 Peckham PH, Keith MW, Freehafer AA (1988) Restoration of functional control by electrical stimulation in the upper extremity of the quadriplegic patient. J Bone Joint Surg 70A: 144-148. 